TITLE:

\title{
Stop model with input-dependent shape function and its identification methods
}

$\operatorname{AUTHOR}(S):$

Matsuo, T; Terada, Y; Shimasaki, M

\section{CITATION:}

Matsuo, T ...[et al]. Stop model with input-dependent shape function and its identification methods. IEEE TRANSACTIONS ON MAGNETICS 2004, 40(4): 1776-1783

\section{ISSUE DATE:}

2004-07

URL:

http://hdl.handle.net/2433/40001

\section{RIGHT:}

(c)2004 IEEE. Personal use of this material is permitted. However, permission to reprint/republish this material for advertising or promotional purposes or for creating new collective works for resale or redistribution to servers or lists, or to reuse any copyrighted component of this work in other works must be obtained from the IEEE. 


\title{
Stop Model With Input-Dependent Shape Function and Its Identification Methods
}

\author{
Tetsuji Matsuo, Member, IEEE, Yasushi Terada, and Masaaki Shimasaki, Member, IEEE
}

\begin{abstract}
We propose an input-dependent shape function for the stop model to remove its property of equal vertical chords regardless of dc bias. We compare several identification methods for the stop model and show that the input-dependent shape function improves the ability to represent the stop model effectively. A least-squares identification method using both symmetric and asymmetric $B-H$ loops achieves the most precise representation of hysteretic characteristics for a silicon steel sheet. However, if only symmetric loops are available for identification, a newly proposed identification method gives more accurate representation than other methods including the least-squares method.
\end{abstract}

Index Terms-Hysteresis, identification, input-dependent shape function, least-squares method, silicon steel sheet, stop model.

\section{INTRODUCTION}

A CCURATE electromagnetic-field analyses for ferromagnetic materials require a precise hysteresis model. For example, the Preisach model [1] and its extended models [2], [3] have been widely used to precisely describe various hysteretic characteristics for arbitrary input sequences. However, in finite-element analyses, the Preisach model poses several obstacles such as a large memory requirement and a high computation cost to calculate the magnetic field $H$ from magnetic flux density $B$ [4]. Because the latter difficulty arises in the electromagnetic-field analysis using the magnetic vector potential formulation, some efficient methods [3]-[5] have been proposed for providing output $H$ from $B$ by the Preisach model.

Some other efficient hysteresis models have been proposed recently, such as the play and stop models [6]-[8], neural-network models [9], [10], and a chemical-reaction model [11]. For example, the play model can be implemented more simply than the Preisach model; moreover, its representation capability has been proven to be equivalent to that of the scalar static Preisach model [7], [12]. The stop model can also be an efficient tool for the electromagnetic-field analysis because: 1) it can directly provide a hysteretic output of $H$ from an input of $B ; 2$ ) it can be implemented as simply as the play model; and 3) it can represent the complex hysteretic characteristics that the Preisach model can. Vector versions of the stop model have been proposed in [6] and [13], [14].

A previous study [15], [16] has proposed an identification method for the scalar stop model from measured $B-H$ loops.

Manuscript received February 3, 2004; revised March 22, 2004. This work was supported in part by the JFE 21st Century Foundation.

The authors are with the Department of Electrical Engineering, the Graduate School of Engineering, Kyoto University, Kyoto 615-8510, Japan (e-mail: tmatsuo@kuee.kyoto-u.ac.jp; simasaki@kuee.kyoto-u.ac.jp; terada@fem.kuee.kyoto-u.ac.jp).

Digital Object Identifier 10.1109/TMAG.2004.828927
It showed that the stop model can simulate an inverse of the Preisach model. However, another study [17] has shown that the stop model cannot sufficiently represent the hysteretic characteristics of a silicon steel sheet because the magnetic characteristics of the silicon steel sheet do not satisfy the property of equal vertical chords regardless of dc bias [15], [16]. Representation by the stop model requires that the property be satisfied.

The present study first proposes an input-dependent shape function for the scalar stop model to remove the property of equal vertical chords regardless of dc bias. Then, several identification methods for the stop model are compared to show that the input-dependent shape function improves the stop model's representation capability effectively.

\section{Stop Model AND Property of EQual Vertical Chords REGARDLESS OF DC BIAS}

The stop model describes a hysteretic relation between $B$ and $H$ as

$$
H=S(B)=\int_{0}^{B_{S}} g\left(\eta, s_{\eta}(B)\right) \mathrm{d} \eta
$$

where $S(B)$ is the hysteretic function represented by the stop model, $B_{\mathrm{S}}$ is the saturation magnetic flux density, $s_{\eta}(B)$ is the stop hysteron operator, $g(\eta, s)$ is the shape function, and $\eta$ is a positive parameter that gives the height of the stop hysteron. It is assumed that the hysteretic function $H=S(B)$ exhibits its hysteretic property when $|B|<B_{\mathrm{S}}$ and becomes a singlevalued function when $|B| \geq B_{\mathrm{S}}$.

The stop hysteron operator of ordinary type is given by

$$
s_{\eta}(B)=\max \left(\min \left(B-B^{0}+s_{\eta}^{0}, \eta\right),-\eta\right)
$$

where $B^{0}$ and $s_{\eta}^{0}$ are the values of $B$ and $s_{\eta}(B)$ at the previous time point. Fig. 1(a) illustrates characteristics of the ordinary stop hysteron operator. However, the function $S(B)$, given by (1) and (2), has a hysteretic property even when $|B| \geq B_{\mathrm{S}}$.

In order for $S(B)$ to become a single-valued function when $|B| \geq B_{\mathrm{S}}$, the operator $s_{\eta}(B)$ is defined as the following:

$$
s_{\eta}(B)=\left\{\begin{array}{ll}
\max \left(\min \left(B-B^{0 *}+s_{\eta}^{0}, \eta\right),-\eta\right) & \left(\eta<B_{\mathrm{S}}\right) \\
B & \left(\eta=B_{\mathrm{S}}\right)
\end{array} .\right.
$$

Therein

$$
B^{0 *}=\max \left(\min \left(B^{0}, B_{\mathrm{S}}\right)-B_{\mathrm{S}}\right) .
$$

Fig. 1(b) illustrates characteristics of this operator $s_{\eta}(B)$ for $\eta<B_{\mathrm{S}}$. 


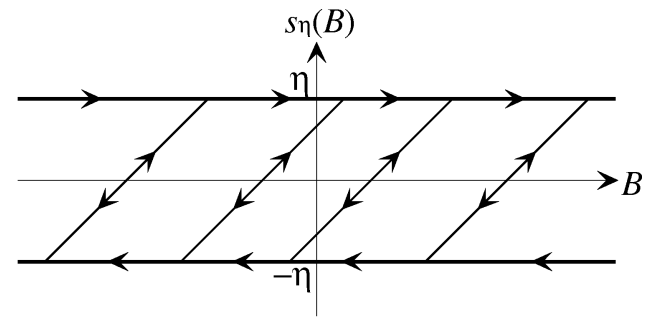

(a)

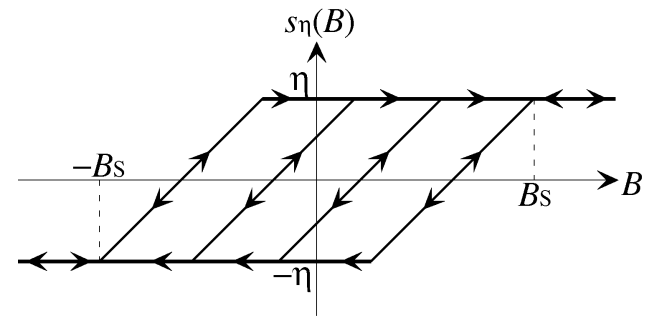

(b)

Fig. 1. Stop hysteron operator: (a) ordinary stop hysteron operator, and (b) stop hysteron operator given by (3) and (4).

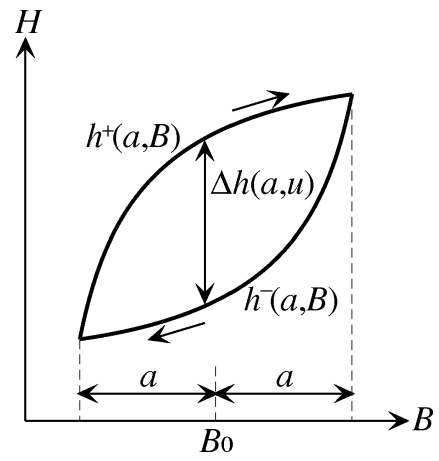

Fig. 2. Ascending and descending curves of $B-H$ loop.

The stop model (1) has the property of equal vertical chords regardless of dc bias [15], [16] as follows. Let $h^{+}(a, B)$ and $h^{-}(a, B)$ be the outputs on ascending and descending curves, respectively, for a back-and-forth input variation given by (5) having amplitude $a\left(0 \leq a \leq B_{\mathrm{S}}\right)$

$$
B=u+B_{0}(-a \leq u \leq a)
$$

where $B_{0}\left(\left|B_{0}\right| \leq\left|B_{\mathrm{S}}-a\right|\right)$ is an arbitrary dc bias (see Fig. 2). It is assumed that the input $B$ has no extrema other than $B_{0} \pm a$. A previous work [15], [16] has shown that the vertical difference $\Delta h$ between $h^{ \pm}\left(a, u+B_{0}\right)$ does not depend on $B_{0}$

$$
h^{+}\left(a, u+B_{0}\right)-h^{-}\left(a, u+B_{0}\right)=\Delta h(a, u) .
$$

In other words, the back-and-forth input variations of the same amplitude produce equal vertical chords regardless of the dc bias.

A previous study [17] has shown that magnetic characteristics of a silicon steel sheet do not satisfy the property of equal vertical chords regardless of dc bias. Accordingly, this stop model cannot represent hysteretic characteristics of the silicon steel sheet accurately.
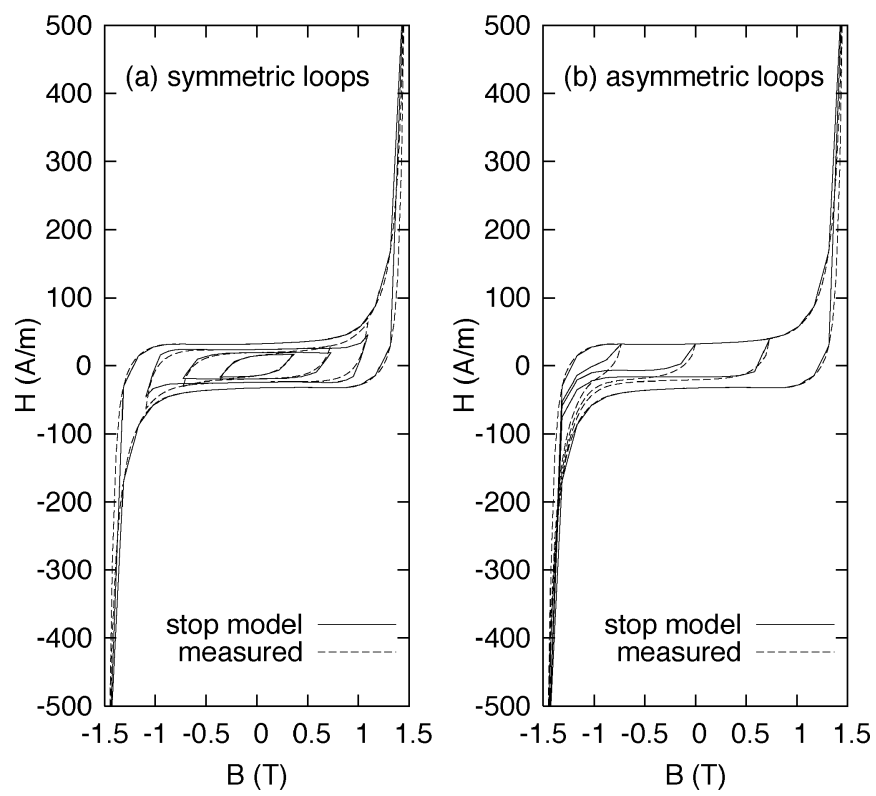

Fig. 3. $B-H$ loops of silicon steel sheet represented by the stop model.
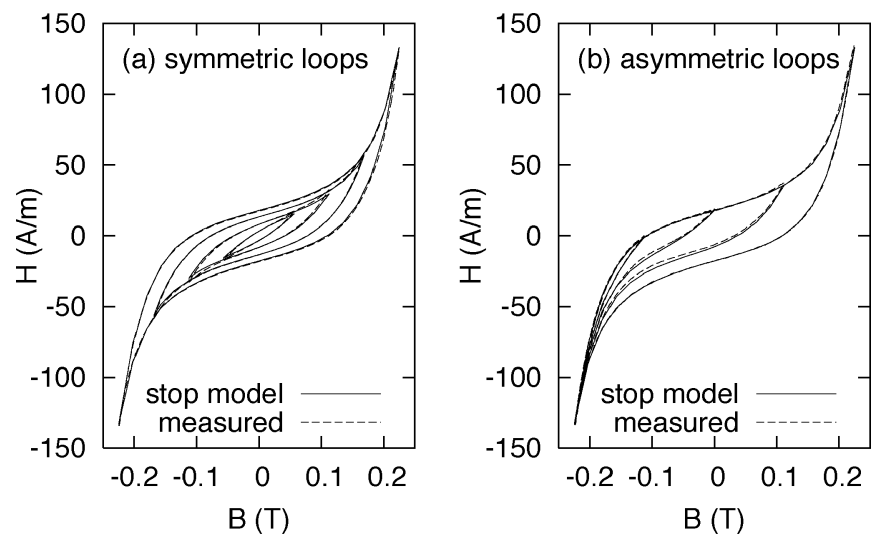

Fig. 4. $\quad B-H$ loops of $\mathrm{Ni}-\mathrm{Zn}$ ferrite ring represented by the stop model.

The present paper examines the representation accuracy of the stop model for two kinds of $B-H$ loops: symmetric loops $\left(B_{0}=0\right)$, and $B-H$ loops that make first-order reversal curves from negative saturation $\left(B_{0}=-B_{\mathrm{S}}+a\right)$. For simplicity, the latter loops are called "asymmetric loops" in this paper.

Symmetric and asymmetric $B-H$ loops of a nonoriented silicon steel sheet (JIS: 50A290) are measured at $1 \mathrm{~Hz}$ by a single sheet tester [18], [19], where the amplitudes of $B-H$ loops are given by $a=k \Delta a$ and $\Delta a=B_{\mathrm{S}} / M(k=1, \ldots, M, M=20$, $B_{\mathrm{S}}=1.46 \mathrm{~T}$ ).

Fig. 3 shows the $B-H$ curves given by the stop model identified from the measured symmetric $B-H$ loops, where the shape function $g(\eta, s)$ is determined by the identification method in [15], [16] with 20 stop hysterons. Fig. 3(b) shows that identification from the symmetric loops results in an inaccurate representation of the asymmetric loops [17].

For comparison, Fig. 4 shows a plot of hysteretic characteristics of a Ni-Zn ferrite ring. The characteristics are represented by the stop model identified from 20 measured symmetric $B-H$ loops of the ferrite ring. Fig. 4 shows that the stop model precisely reconstructs the hysteretic characteristics. 


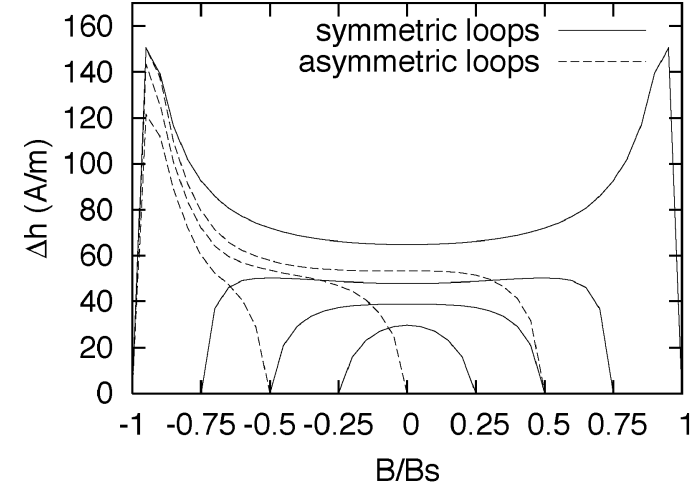

(a)

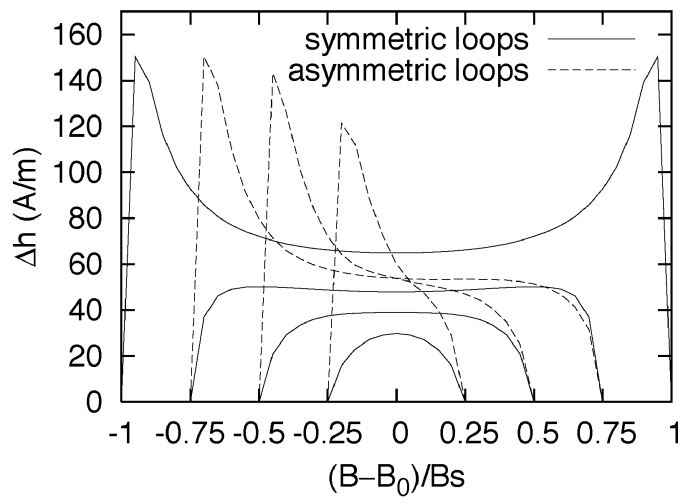

(b)

Fig. 5. Vertical chords $\Delta h$ of the silicon steel sheet: (a) $B / B_{\mathrm{S}}$ versus $\Delta h$, and (b) $\left(B-B_{0}\right) / B_{\mathrm{S}}$ versus $\Delta h$.

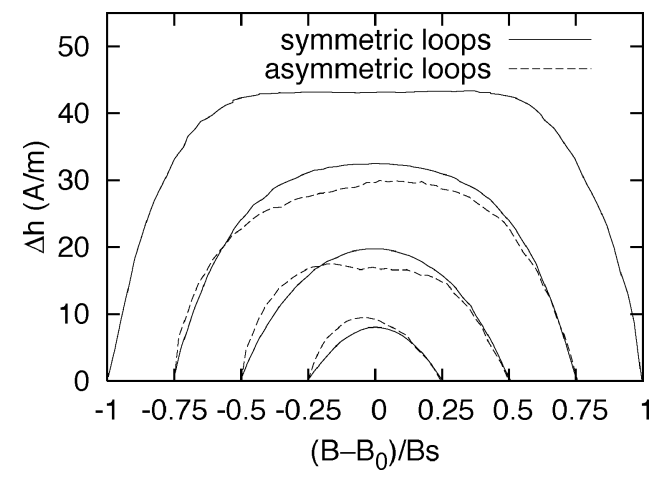

Fig. 6. Vertical chords $\Delta h$ of the $\mathrm{Ni}-\mathrm{Zn}$ ferrite ring.

Figs. 5 and 6 show the vertical chords of $B-H$ loops of the silicon steel sheet and the $\mathrm{Ni}-\mathrm{Zn}$ ferrite ring, respectively, where $\Delta h$ of the symmetric and asymmetric loops are compared. Fig. 6 shows that hysteretic characteristics of the ferrite have approximate properties of equal vertical chords regardless of dc bias, which engenders agreement between the measured and simulated $B-H$ loops. In contrast, as seen in Fig. 5, vertical chords of asymmetric loops of the silicon steel sheet differ considerably from those of symmetric loops. The vertical chords are large near $B=B_{\mathrm{S}}$, whereas they are small near $B=0$. This strong dependency of vertical chords on the dc bias causes a large discrepancy between the measured and simulated asymmetric loops.

\section{Stop Model With InPut-DePEndent Shape Function}

The present study induces an input-dependent shape function for the stop model to remove the property of equal vertical chords regardless of dc bias

$$
H=S(B)=\int_{0}^{B_{\mathrm{S}}} g\left(\eta, s_{\eta}(B), B\right) \mathrm{d} \eta .
$$

Therein, $g(\eta, s, B)$ is the input-dependent shape function.

For simplicity, the shape function is set in this study as

$$
g(\eta, s, B)=g_{0}(\eta, s) w(B)
$$

where $w(B)$ is called the weighting function. Equations (7) and (8) imply that

$$
S(B)=w(B) S_{0}(B)
$$

where $S_{0}(B)$ is the stop model having the input-independent shape function $g_{0}$, which is given by

$$
S_{0}(B)=\int_{0}^{B_{\mathrm{S}}} g_{0}\left(\eta, s_{\eta}(B)\right) \mathrm{d} \eta .
$$

The vertical chord of this stop model $S(B)$ depends on the dc bias because it is given by

$$
\Delta h(a, u)=w\left(u+B_{0}\right) \Delta h_{0}(a, u)
$$

where $\Delta h_{0}$ is the vertical chord of $S_{0}(B)$. Equation (11) implies that $\Delta h(a, u)$ does not depend on the position of $B-H$ loops along the $H$ direction if the amplitude and dc bias of the back-and-forth input variations are identical. Therefore, this stop model has the property of equal vertical chords in the same manner as the nonlinear (input-dependent) Preisach model proposed by Mayergoyz [2]. Because $S(B) / w(B)$ represents the stop model having an input-independent shape function, $H(B) / w(B)$ should have the property of equal vertical chords regardless of dc bias. Thereby, it is represented precisely by this stop model.

The present study uses the fact that the vertical chords are large where $\Delta h\left(B_{\mathrm{S}}, B\right)$ is large, i.e., the vertical chords are large where the vertical chord of the major $B-H$ loop is large, as seen in Fig. 5(a). This fact implies that the dependency of the vertical chords on the dc bias is evaluated roughly from $\Delta h\left(B_{\mathrm{S}}, B\right)$ because the major loop has the maximum vertical chord among all the $B-H$ loops. The weighting function is set accordingly as

$$
w(B)=\Delta h\left(B_{\mathrm{S}}, B\right)=h_{M}^{+}(B)-h_{M}^{-}(B)
$$

where $h_{M}^{ \pm}(B)$ denotes ascending and descending curves of the measured major hysteresis loop of $H(B)$, respectively. Hysteretic characteristics of $H(B) / w(B)$ with (12) are expected to roughly satisfy the property of equal vertical chords regardless of dc bias because its major $B-H$ loop has a constant vertical 


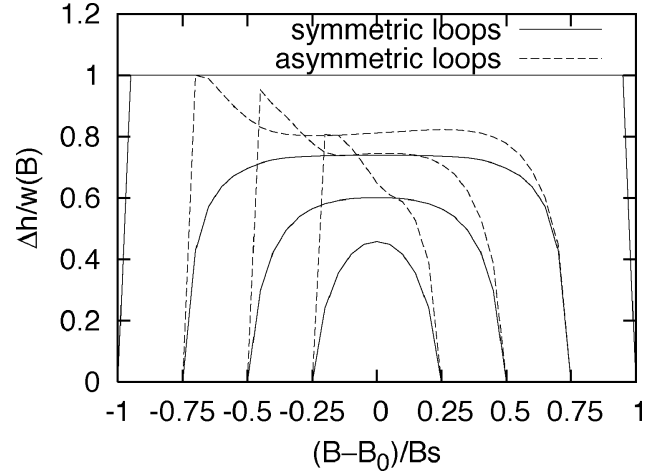

Fig. 7. Vertical chords $\Delta h / w(B)$.
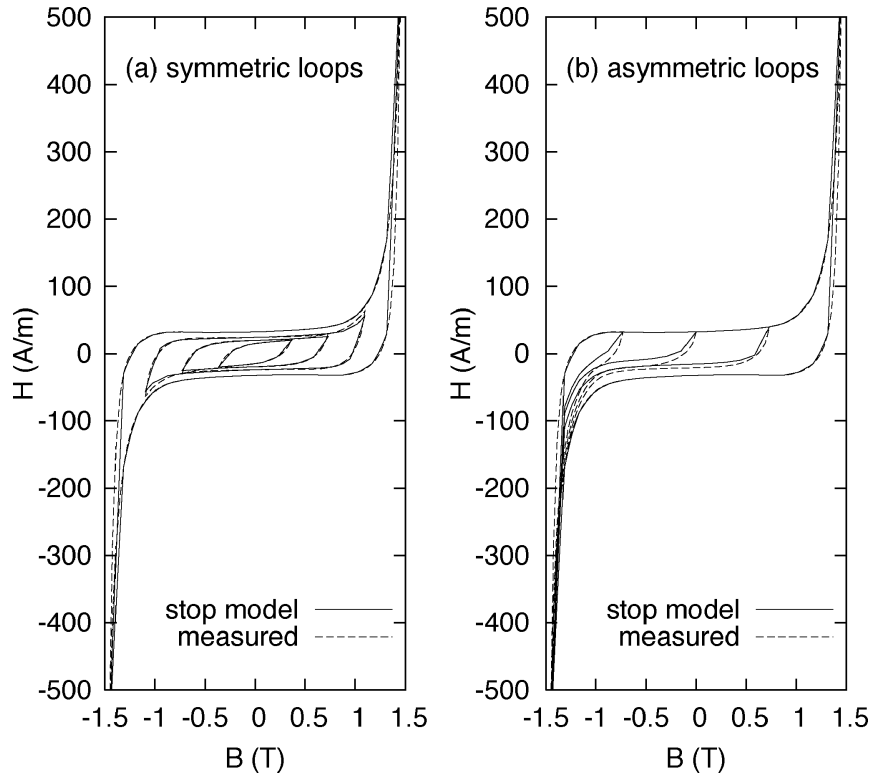

Fig. 8. Stop model using input-dependent shape function.

chord independently from $B$. However, the weighting function $w(B)$ given by (12) becomes 0 at $B= \pm B_{\mathrm{S}}$. Accordingly, it should be modified near $B= \pm B_{\mathrm{S}}$, as shown in Section IV-B.

Vertical chords $\Delta h / w(B)$ using (12) are plotted in Fig. 7, where the discrepancy of the vertical chords between the symmetric and asymmetric loops becomes small compared with Fig. 5(b).

The stop model using the weighting function (12) is identified from the 20 symmetric loops of the silicon steel sheet, where $g_{0}(\eta, s)$ is determined by the identification method in [15], [16]. Fig. 8 shows the $B-H$ loops given by the stop model. Comparison of Fig. 8 with Fig. 3 shows that the weighting function (12) improves the representation of hysteretic characteristics.

\section{NEW IdENTIFICATION Method}

\section{A. Identification Method Using Loop Shapes}

The identification method proposed in [15], [16] uses only loop widths along the $H$ direction and does not use shapes of $B-H$ loops. The present paper proposes a new identification method that uses shapes of symmetric $B-H$ loops.
The periodic input (5) for the symmetric loop $\left(B_{0}=0\right)$ induces the response of the stop hysteron as

$$
s_{\eta}(B)= \begin{cases}B & (\eta \geq a) \\ \min (B-\eta+a, \eta) & (\eta<a, \text { when increasing }) \\ \max (B+\eta-a,-\eta) & (\eta<a, \text { when decreasing })\end{cases}
$$

By setting $\kappa(\eta, s)=\partial g_{0}(\eta, s) / \partial s\left(-\eta \leq s \leq \eta, \eta \leq B_{\mathrm{S}}\right)$, the shape function $g_{0}$ is written as

$$
g_{0}\left(\eta, s_{\eta}\right)=\int_{-\eta}^{s_{\eta}} \kappa(\eta, s) \mathrm{d} s+g_{0}(\eta,-\eta) .
$$

From (7), (8), and (14), $S(B) / w(B)$ is written as

$$
\frac{S(B)}{w(B)}=\int_{0}^{B_{\mathrm{S}}} \int_{-\eta}^{s_{\eta}(B)} \kappa(\eta, s) \mathrm{d} s \mathrm{~d} \eta+\frac{-H_{\mathrm{S}}}{w\left(-B_{\mathrm{S}}\right)}
$$

where $-H_{\mathrm{S}}=H\left(-B_{\mathrm{S}}\right)$ is represented as

$$
-H_{\mathrm{S}}=w\left(-B_{\mathrm{S}}\right) \int_{0}^{B_{\mathrm{S}}} g_{0}(\eta,-\eta) \mathrm{d} \eta .
$$

From (13) and (15), the descending curve of the symmetric $B-H$ loop having amplitude $a, h^{-}(a, B)(-a \leq B \leq a)$, is given by

$$
\begin{aligned}
& \frac{h^{-}(a, B)}{w(B)}-\frac{-H_{\mathrm{S}}}{w\left(-B_{\mathrm{S}}\right)}=D(a, B)=\hat{D}(c, B) \\
& D(a, B)=\int_{\frac{a-B}{2}}^{B_{\mathrm{S}}} \int_{-\eta}^{\min (B, B+\eta-a)} \kappa(\eta, s) \mathrm{d} s \mathrm{~d} \eta \\
& \hat{D}(c, B)=\int_{c}^{2 B_{\mathrm{S}}} \int_{-\frac{t}{2}}^{\min \left(B,-t+B_{\mathrm{S}}\right)} \hat{\kappa}(t, s) \mathrm{d} s \mathrm{~d} t
\end{aligned}
$$

where $c=a-B, t=\eta-s$, and $\hat{\kappa}(t, s)=\kappa(\eta, s)(-t / 2 \leq s \leq$ $\left.-t+B_{\mathrm{S}}, 0 \leq t\right)$. The integral domains for $D(a, B)$ and $\hat{D}(c, B)$ are illustrated in Fig. 9(a) and (b), respectively. Equation (19) implies that

$$
\kappa(a, B)=\hat{\kappa}(c, B)=-\frac{\partial^{2} \hat{D}(c, B)}{\partial c \partial B} .
$$

\section{B. Numerical Implementation of Identification Method}

This new identification method can be implemented as described below.

The stop model (7) using (8) in the discretized form is written as

$$
S(B)=w(B) \sum_{n=1}^{N_{\mathrm{s}}} \tilde{g}_{n}\left(s_{\eta_{n}}(B)\right)
$$

where $\eta_{n}=n \Delta \eta, \Delta \eta=B_{\mathrm{S}} / N_{\mathrm{s}}\left(n=1, \ldots, N_{\mathrm{s}}, N_{\mathrm{s}}\right.$ : the number of stop hysterons) and $\tilde{g}_{n}$ is an input-independent shape 


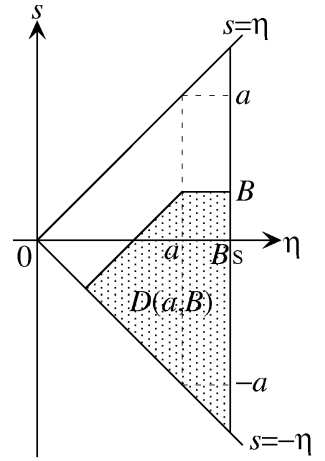

(a)

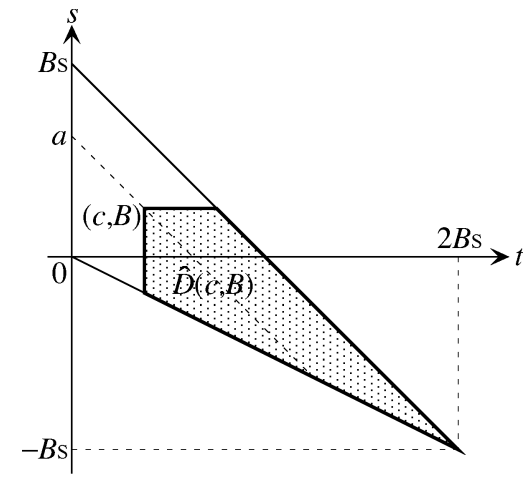

(b)

Fig. 9. Integral domains: (a) integral domain for $D(a, B)$, and (b) integral domain for $\hat{D}(c, B)$.

function for $s_{\eta_{n}}(B)$. The shape function is assumed to be piecewise linear as

$$
\begin{aligned}
& \tilde{g}_{n}(s)=\tilde{g}_{n}\left(s_{m-1}\right)+\frac{\tilde{\kappa}_{n, m-\frac{1}{2}}\left(s-s_{m-1}\right)}{\Delta s} \\
&\left(s_{m-1} \leq s \leq s_{m}, m=-n+1, \ldots, n\right)
\end{aligned}
$$

where $s_{m}=m \Delta s\left(m=-N_{\mathrm{s}}, \ldots, N_{\mathrm{s}}\right), \Delta s=B_{\mathrm{S}} / N_{\mathrm{s}}$, and $\tilde{\kappa}_{n, m-(1 / 2)}=\tilde{g}_{n}\left(s_{m}\right)-\tilde{g}_{n}\left(s_{m-1}\right)$. It is noteworthy that the piecewise linear shape function $\tilde{g}_{n}$ has $(2 n+1)$ nodes, whereas the identification method in [15], [16] uses a shape function having only $(n+1)$ nodes.

Measured descending curves of symmetric loops, $h^{-}\left(\eta_{k}, B\right)$, $\left(k=1, \ldots, N_{\mathrm{s}}\right)$ are used for identification as follows. For inputs $B=b_{j}\left(b_{j}=j B_{\mathrm{S}} / N_{\mathrm{s}}, j=-k, \ldots, k\right), h^{-}\left(\eta_{k}, b_{j}\right)$ is given as

$$
\begin{gathered}
\frac{h^{-}\left(\eta_{k}, b_{j}\right)}{w\left(b_{j}\right)}-\frac{-H_{\mathrm{S}}}{w\left(-B_{\mathrm{S}}\right)}=\sum_{n=\left\lceil\frac{(k-j+1)}{2}\right\rceil}^{N_{\mathrm{s}}} \sum_{m=-n+1}^{\min (j, j+n-k)} \tilde{\kappa}_{n, m-\frac{1}{2}} \\
-H_{\mathrm{S}}=w\left(-B_{\mathrm{S}}\right) \sum_{n=1}^{N_{\mathrm{S}}} \tilde{g}_{n}\left(-\eta_{n}\right)
\end{gathered}
$$

where $\lceil x\rceil$ indicates the smallest integer not less than $x$. Equation (23) is the discretized description of (17). Summation in (23) is performed in the hatched region in Fig. 10(a), which cor-

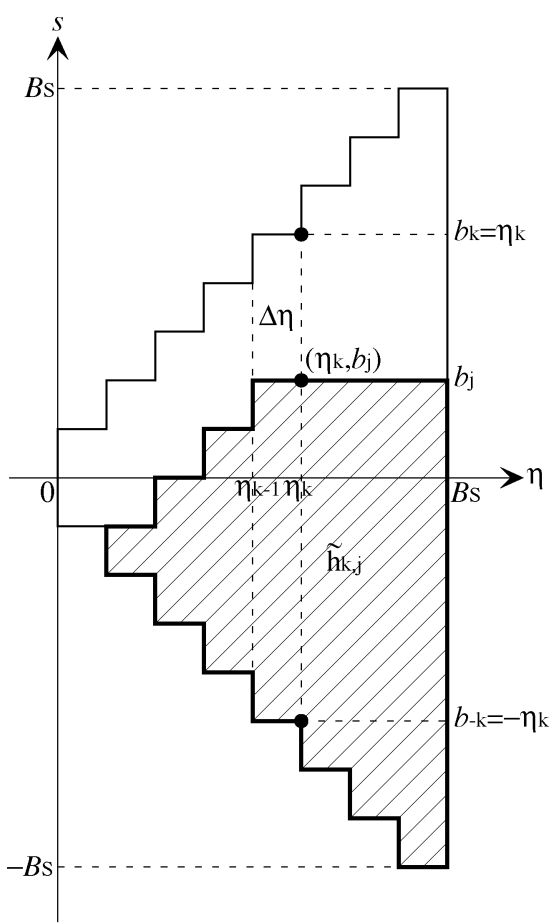

(a)

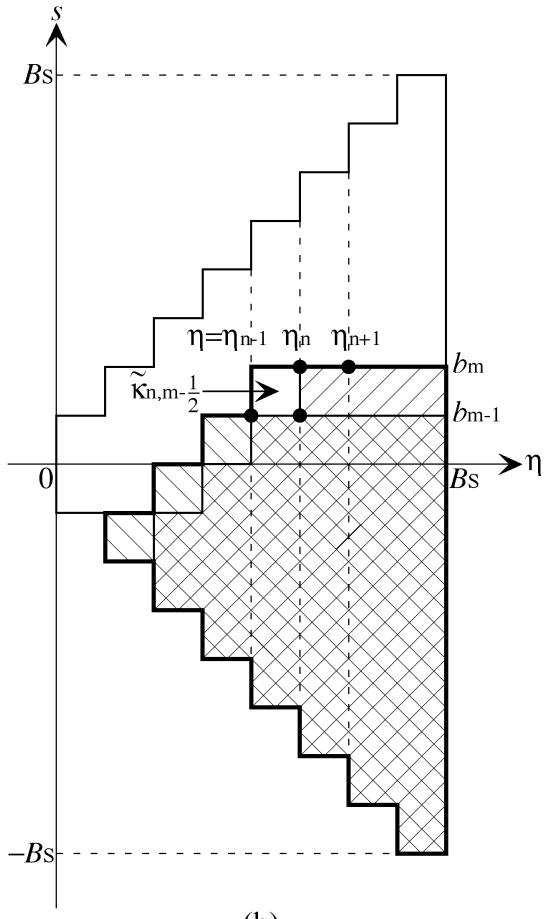

(b)

Fig. 10. Calculations of (23) and (26): (a) $\tilde{h}_{k, j}^{-}$, and (b) $\tilde{\kappa}_{n, m-(1 / 2)}$.

responds to Fig. 9(a). The descending curves yield $\tilde{\kappa}_{n, m-(1 / 2)}$ $\left(n=1, \ldots, N_{\mathrm{s}}, m=-n+1, \ldots, n\right)$ as the following:

$$
\begin{aligned}
\tilde{\kappa}_{n, m-\frac{1}{2}} & =\tilde{h}_{n, m}^{-}-\tilde{h}_{n-1, m-1}^{-}-\tilde{h}_{n+1, m}^{-}+\tilde{h}_{n, m-1}^{-} \\
\tilde{h}_{n, m}^{-} & =\frac{h^{-}\left(\eta_{n}, b_{m}\right)}{w\left(b_{m}\right)}\left(n=1, \ldots, N_{\mathrm{s}}, m=-n+1, \ldots, n\right)
\end{aligned}
$$

$\tilde{h}_{N_{\mathrm{s}},-N_{\mathrm{s}}}^{-}=\frac{h^{-}\left(\eta_{N_{\mathrm{s}}}, b_{-N_{\mathrm{s}}}\right)}{w\left(b_{-N_{\mathrm{s}}}\right)}$ 

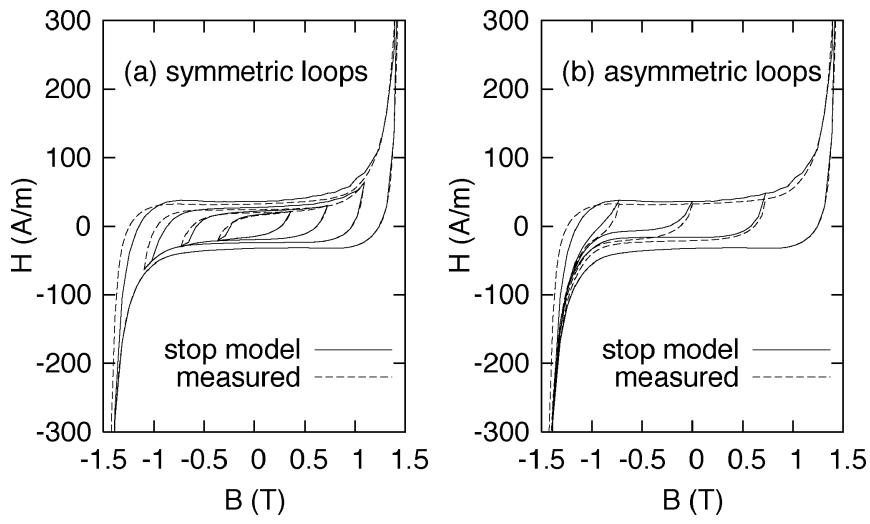

Fig. 11. Stop model identified by the proposed method without symmetrization.

$$
\begin{gathered}
\tilde{h}_{n,-n}^{-}=\tilde{h}_{n+1,-n}^{-}, \tilde{h}_{n,-n-1}^{-}=\tilde{h}_{\min \left(n+2, N_{\mathrm{s}}\right),-n-1}^{-} \\
\left(n=0, \ldots, N_{\mathrm{s}}-1\right) \\
\tilde{h}_{N_{\mathrm{s}}+1, m}^{-}=\tilde{h}_{N_{\mathrm{s}}, m-1}^{-}\left(m=-N_{\mathrm{s}}+1, \ldots, N_{\mathrm{s}}\right) .
\end{gathered}
$$

The calculation of (25) is illustrated in Fig. 10(b).

The weighting function $w(B)$ is also assumed to be piecewise linear as

$$
w(B)=w_{j-1}+\frac{w_{j}-w_{j-1}}{b_{j}-b_{j-1}}\left(B-b_{j-1}\right) \quad\left(b_{j-1} \leq B \leq b_{j}\right)
$$

where

$$
\begin{gathered}
w_{j}=h_{M}^{+}\left(b_{j}\right)-h_{M}^{-}\left(b_{j}\right) \quad\left(j=-N_{\mathrm{s}}+1, \ldots, N_{\mathrm{s}}-1\right) \\
w_{ \pm N_{\mathrm{s}}}=2 w_{ \pm\left(N_{\mathrm{s}}-1\right)}-w_{ \pm\left(N_{\mathrm{s}}-2\right)} .
\end{gathered}
$$

Fig. 11 shows hysteretic characteristics identified by the above method using (30)-(32) with 20 hysterons, where the ascending curves are not precisely represented. Fig. 12 shows symmetrized characteristics of this stop model that are given by symmetrizing the shape function as

$$
\tilde{\kappa}_{n, m-\frac{1}{2}} \leftarrow \frac{\left(\tilde{\kappa}_{n, m-\frac{1}{2}}+\tilde{\kappa}_{n,-m+\frac{1}{2}}\right)}{2} .
$$

Fig. 12 shows that the symmetrized model yields smoother $B-H$ curves than those in Fig. 8 because of the increase in the number of nodes of the piecewise linear shape functions.

\section{IDENTIFICATION BY THE LEAST-SQUARES METHOD}

A least-squares method can be applied for identification of the stop model [7], [20]. The least-squares method determines the shape function $g_{0}$ so as to minimize

$$
\sum_{i}^{I}\left|H\left(B_{i}\right)-S\left(B_{i}\right)\right|^{2}
$$

where $B_{i}$ ( $i=1,2, \ldots, I, I$ : the number of input points) is an input sequence and $H\left(B_{i}\right)$ is the corresponding sequence of the measured magnetic field. It has been shown in [20] that the height difference $\Delta \eta$ of the stop hysterons should not be smaller
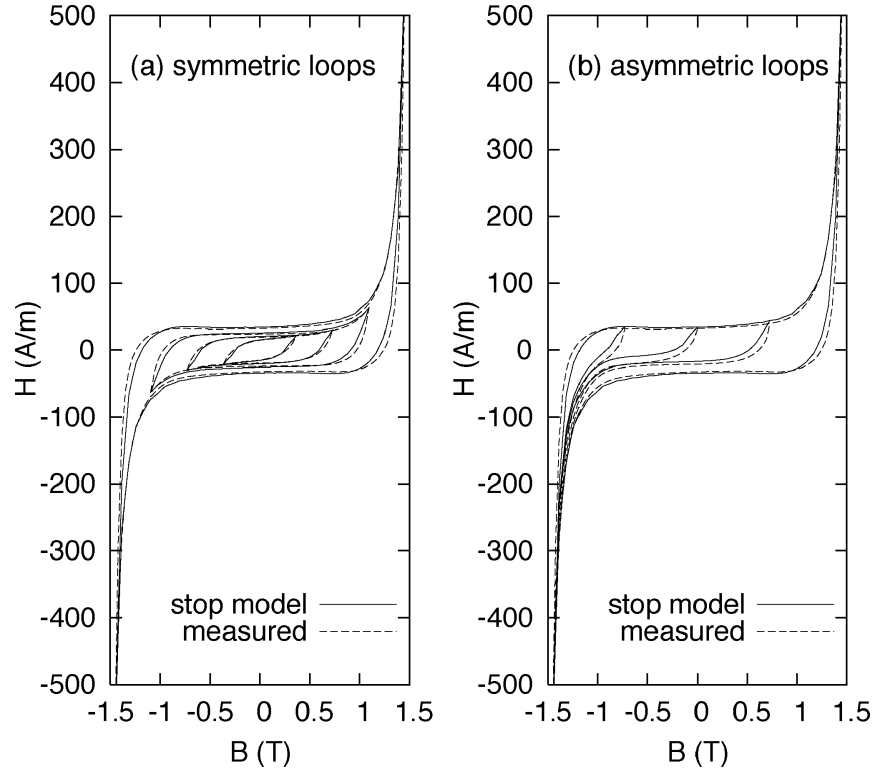

Fig. 12. Stop model identified by the proposed method and symmetrized.
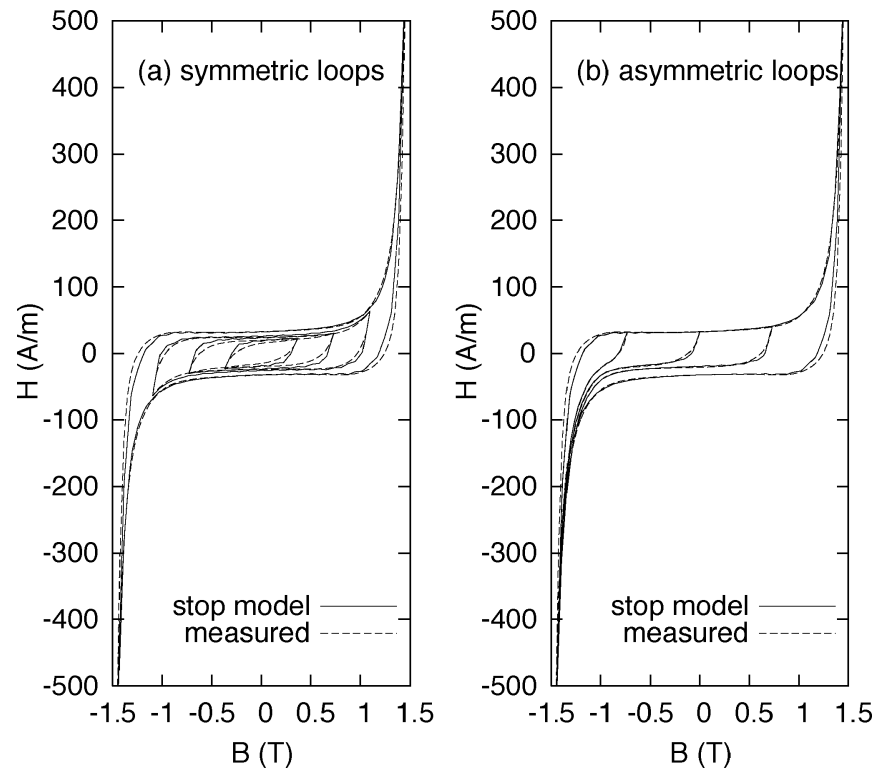

Fig. 13. Stop model identified by the least-squares method.

than the amplitude difference $\Delta a$ of the $B-H$ loops used for identification (see the Appendix).

The discretized stop model (21) using (22) is identified by the least-squares method using (12) [i.e., (30)-(32)] with 20 hysterons, where the input sequence is given by both the symmetric and asymmetric $B-H$ loops: 1$)$ the ascending and descending curves of symmetric loops; 2) the first-order reversal curves from the negative saturation; and also 3 ) the first-order reversal curves from the positive saturation. The second and third types of curves are given from the descending curves of the asymmetric loops as $\pm\left(B, h^{-}(a, B)\right)\left(-B_{\mathrm{S}} \leq B \leq-B_{\mathrm{S}}+2 a\right)$. The third curves are required for symmetry.

The hysteretic characteristics identified by the least-squares method above are shown in Fig. 13. That figure shows that the least-squares method achieves a more precise representation than do the other identification methods as shown in Figs. 8 and 
TABLE I

Average REPRESENTATION ERRORs (\%) GIVEN By tHE FIVE IDENTIFICATION Methods Using $w(B)=1$

\begin{tabular}{c||c|c|c|c|c} 
method & $(\mathrm{a})$ & $(\mathrm{b})$ & $(\mathrm{c})$ & $(\mathrm{d})$ & $(\mathrm{e})$ \\
\hline symmetric loops & 2.40 & 3.19 & 1.36 & 1.43 & 0.64 \\
\hline asymmetric loops & 4.95 & 4.27 & 3.21 & 1.50 & 5.21 \\
\hline total error & 3.89 & 3.77 & 2.47 & 1.47 & 3.71 \\
\hline
\end{tabular}

TABLE II

Average RePresentation ERRoRs (\%) Given by the Five IDENTIFICATION Methods Using THE $w(B)$ OF (12)

\begin{tabular}{c||c|c|c|c|c} 
method & $(\mathrm{a})$ & $(\mathrm{b})$ & $(\mathrm{c})$ & $(\mathrm{d})$ & $(\mathrm{e})$ \\
\hline symmetric loops & 2.05 & 2.18 & 1.10 & 0.99 & 0.35 \\
\hline asymmetric loops & 3.76 & 3.23 & 2.42 & 0.88 & 4.12 \\
\hline total error & 3.03 & 2.80 & 1.88 & 0.94 & 2.92 \\
\hline
\end{tabular}

12. However, the small discrepancy between the measured $H$ and the simulated one remains because the weighting function (12) is so simple that $H(B) / w(B)$ does not completely satisfy the property of equal vertical chords regardless of dc bias. Future work will address further improvement of the representation accuracy to find a more effective weighting function.

\section{COMPARISON OF IDENTIFICATION METHODS}

The identification methods (below) examined in the previous sections are compared, where the weighting function (12) [i.e., (30)-(32)] is used for the input-dependent shape function:

(a) an identification method using loop widths of symmetric loops (corresponding to Figs. 3 and 8);

(b) an identification method using loop widths of asymmetric loops with symmetrization of the shape function;

(c) an identification method using descending curves of symmetric loops with symmetrization of the shape function (corresponding to Fig. 12);

(d) a least-squares method (corresponding to Fig. 13);

(e) a least-squares method using only the symmetric loops for identification.

Tables I and II list the average discrepancies between the measured $H(H(B))$ and the simulated one $(S(B))$. They are given by the five identification methods listed above using input-independent and input-dependent shape functions, respectively. The discrepancy $(\%)$ is given as the ratio of the root-mean-square of $\left|H\left(B_{i}\right)-S\left(B_{i}\right)\right|$ to the $H_{\mathrm{S}}=601 \mathrm{~A} / \mathrm{m}$, where the input sequence of $B$ is given by the symmetric and asymmetric loops.

Tables I and II show that the least-squares method (d) using symmetric and asymmetric loops achieves the most precise representation of both the symmetric and asymmetric loops, whereas identification by the least-squares method (e) using only the symmetric loops results in poor representation accuracy of asymmetric loops. The newly proposed method (c) gives the smallest total representation error among identification methods (a), (c), and (e), which use only the symmetric loops. Comparison of Tables I and II shows that the simple weighting function (12) reduces the representation error effectively.

Next, two $B-H$ curves shown in Fig. 14 are simulated by the stop model identified by methods (a)-(d) using the weighting function (12) to examine the response to input sequences other
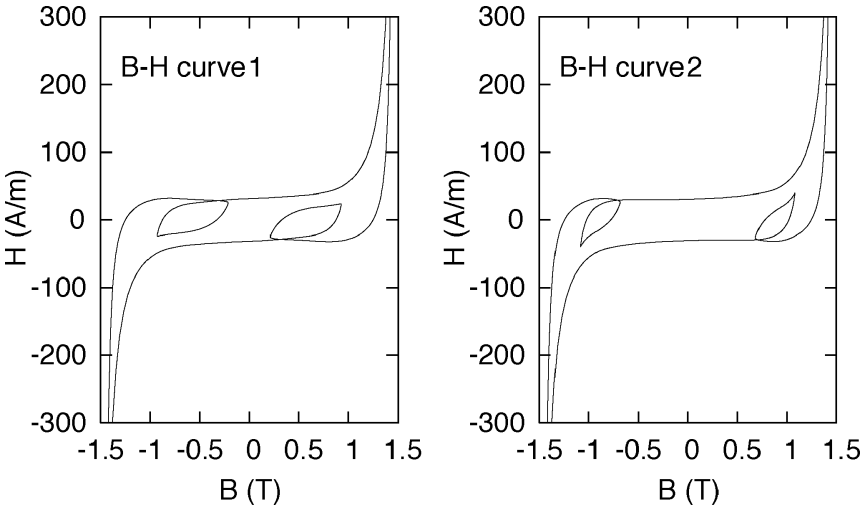

Fig. 14. Two $B-H$ curves having minor loops.
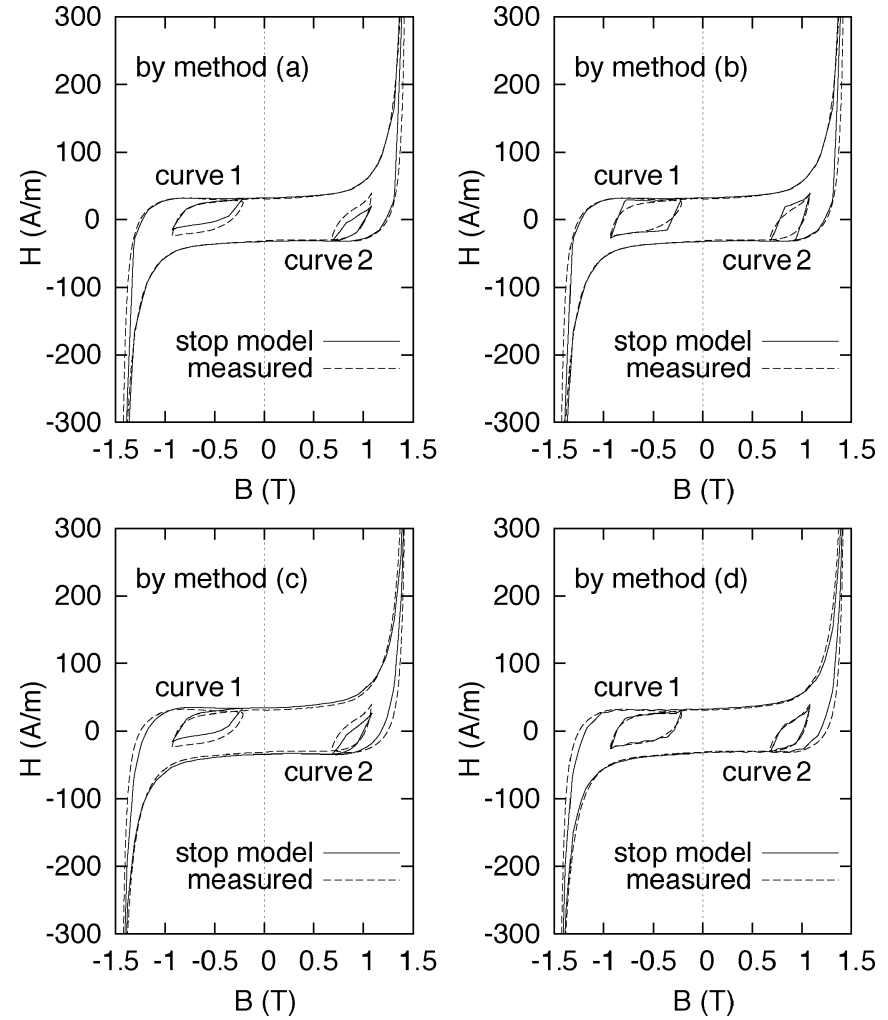

Fig. 15. $B-H$ curve having minor loops obtained from the four identification methods.

than those used for identification. Obtained $B-H$ curves are plotted in Fig. 15, where the least-squares method achieves the most accurate representation.

\section{CONCLUDING REMARKS}

The present paper proposed an input-dependent shape function for the stop model to remove the property of equal vertical chords regardless of dc bias. The present paper also proposed a new identification method for the stop model, which uses the descending curves of measured symmetric loops.

Comparison of results from several identification methods for the stop model shows:

- the input-dependent shape function using a simple weighting function effectively reduces the representation error; 

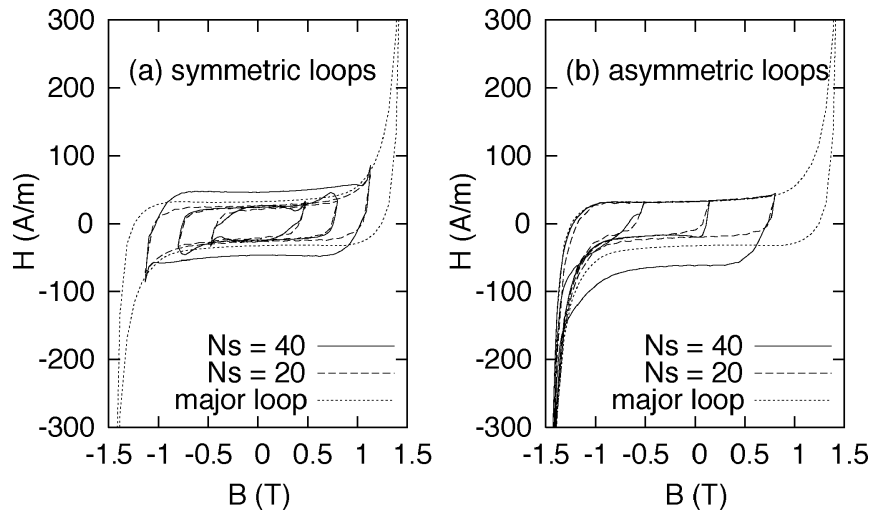

Fig. 16. $B-H$ loops that are not used in the identification.

- the least-squares method achieves the most accurate representation of both symmetric and asymmetric loops;

- if only the symmetric loops are available for identification, the newly proposed identification method gives a smaller representation error than the other methods, including the least-squares method.

\section{APPENDIX}

For convenience of the identification procedure, it is assumed that

$$
\begin{aligned}
\tilde{g}_{n}\left(s_{-n}\right) & =0, \quad\left(n=1, \ldots, N_{\mathrm{s}}-1\right) \\
\tilde{g}_{N_{\mathrm{S}}}\left(s_{-N_{\mathrm{S}}}\right) & =\frac{-H_{\mathrm{S}}}{w\left(-B_{\mathrm{S}}\right)} .
\end{aligned}
$$

A given input sequence of $B_{i}(i=1, \ldots$,$) uniquely determines$ the corresponding values of $s_{\eta_{n}}\left(B_{i}\right)\left(n=1, \ldots, N_{\mathrm{s}}\right)$. Therefore, (21), (22), (35), and (36) lead to the corresponding output sequence $S\left(B_{i}\right)$ written in the form of the sum of $-H_{\mathrm{S}}$ and a linear combination of $\kappa_{n, m}$, as

$$
S\left(B_{i}\right)=\boldsymbol{a}_{i} \cdot \boldsymbol{\kappa}-H_{\mathrm{S}}
$$

where $\boldsymbol{\kappa}=\left(\kappa_{1,-(1 / 2)}, \kappa_{1,(1 / 2)}, \ldots, \kappa_{N_{\mathrm{s}}, N_{\mathrm{s}}-(1 / 2)}\right)^{T}\left({ }^{T}\right.$ : transpose), and $\boldsymbol{a}_{i}$ is the coefficient vector of the linear combination.

Identification by the least-squares method yields $\kappa$ so as to minimize

$$
\|A \kappa-b\|^{2}
$$

where $\boldsymbol{A}$ is the coefficient matrix of which the $i$ th row is $\boldsymbol{a}_{i}^{T}$ and $\boldsymbol{b}$ is the vector of which the $i$ th component is $H\left(B_{i}\right)+H_{\mathrm{S}}$.

Fig. 16 shows simulated $B-H$ loops that are not used for identification by the least-squares method, where the solid lines are given by the stop model with 40 hysterons and the dashed lines are with 20 hysterons. Fig. 16 shows that the stop model using 40 hysterons yields unnatural $B-H$ loops because of over- fitting to the $B-H$ loops used for identification. A previous study [15], [16] has shown that a periodic input of $B$ with amplitude $a$ leads to a hysteretic response of the stop hysterons having height $\eta<a$, and a reversible response of those with $\eta \geq a$. This implies that the amplitude difference $\Delta a$ of periodic inputs of $B$ should not be larger than $\Delta \eta$ [20]. This size limit would allow the identification to distinguish the responses of hysterons having heights $k \Delta \eta(k=1,2, \ldots)$.

\section{REFERENCES}

[1] F. Preisach, "Über die Magnetische Nachwirkung," Zeitschrift Phys., vol. 94 , pp. 277-302, 1935 .

[2] I. D. Mayergoyz, Mathematical Models of Hysteresis. New York: Springer-Verlag, 1991.

[3] E. Della Torre, Magnetic Hysteresis. Piscataway, NJ: IEEE Press, 1999.

[4] N. Takahashi, S. Miyabara, and K. Fujiwara, "Problems in practical finite element analysis using Preisach hysteresis model," IEEE Trans. Magn., vol. 35, pp. 1243-1246, May 1999.

[5] G. S. Park, S. Y. Hahn, K. S. Lee, and H. K. Jung, "Implementation of hysteresis characteristics using the Preisach model with M-B variables," IEEE Trans. Magn., vol. 29, pp. 1542-1545, Mar. 1993.

[6] M. A. Krasnosel'skii and A. V. Pokrovskii, Systems With Hysteresis. Berlin, Germany: Springer-Verlag, 1989.

[7] S. Bobbio, G. Miano, C. Serpico, and C. Visone, "Models of magnetic hysteresis based on play and stop hysterons," IEEE Trans. Magn., vol. 33, pp. 4417-4426, Nov. 1997.

[8] S. Cincotti and I. Daneri, "A nonlinear circuit model of hysteresis," IEEE Trans. Magn., vol. 35, pp. 1247-1250, May 1999.

[9] C. Serpico and C. Visone, "Magnetic hysteresis modeling via feed-forward neural networks," IEEE Trans. Magn., vol. 34, pp. 623-628, May 1998.

[10] D. Makaveev, L. Dupré, M. De Wulf, and J. Melkebeek, "Combined Preisach-Mayergoyz-neural-network vector hysteresis model for electrical steel sheets," J. Appl. Phys., vol. 93, pp. 6638-6640, 2003.

[11] A. Nourdine, G. Meunier, and A. Kedous-Lebouc, "Numerical computation of a vectorial hysteresis $H(B)$ magnetization law," IEEE Trans. Magn., vol. 39, pp. 1393-1396, May 2003.

[12] M. Brokate, "Some mathematical properties of the Preisach model for hysteresis," IEEE Trans. Magn., vol. 25, pp. 2922-2924, July 1989.

[13] T. Matsuo, Y. Osaka, and M. Shimasaki, "Eddy-current analysis using vector hysteresis models with play and stop hysterons," IEEE Trans. Magn., vol. 36, pp. 1172-1177, July 2000.

[14] — "Correction to 'Eddy-current analysis using vector hysteresis models with play and stop hysterons'," IEEE Trans. Magn., vol. 36, pp. 4050-4051, Nov. 2000.

[15] T. Matsuo and M. Shimasaki, "Isotropic vector hysteresis represented by superposition of stop hysteron models," IEEE Trans. Magn., vol. 37 pp. 3357-3361, Sept. 2001.

[16] - "Correction to: 'Isotropic vector hysteresis represented by superposition of stop hysteron models'," IEEE Trans. Magn., vol. 37, p. 3995, Nov. 2001.

[17] T. Matsuo, D. Shimode, Y. Terada, and M. Shimasaki, "Application of stop and play models to the representation of magnetic characteristics of silicon steel sheet," IEEE Trans. Magn., vol. 39, pp. 1361-1364, May 2003.

[18] H. Nishimoto, S. Miyabara, M. Nakano, K. Fujiwara, and N. Takahashi, "Evaluation method of magnetic properties of electrical steel sheets by using the Preisach model," in Papers Tech. Meeting IEE Jpn., 1997, MAG-97-178, pp. 41-46.

[19] H. Nishimoto, M. Nakano, K. Fujiwara, and N. Takahashi, "Effect of frequency on magnetic properties," in Papers Tech. Meeting IEE Jpn., 1998, MAG-98-56, pp. 21-26.

[20] T. Matsuo, K. Ando, Y. Terada, and M. Shimasaki, "A study of representation of hysteretic characteristics by stop and play models," Trans. Inst. Elect. Eng. Jpn. EIS, vol. 123, pp. 1958-1963, 2003. 